\title{
Challenges in the Manufacturing and Operations of Graphene
}

\author{
Y. Wang \\ School of materials \\ University of Manchester \\ Manchester, UK \\ yi.wang-2@manchester.ac.uk
}

\begin{abstract}
Graphene is a two-dimensional, atomic-scale, honey-comb lattice in which one atom forms each vertex. It is the basic structural element of other allotropes, including graphite, charcoal, carbon nanotubes and fullerenes. It can also be considered as an indefinitely large aromatic molecule, the ultimate case of the family of flat polycyclic aromatic hydrocarbons. Graphene has many extraordinary properties. It is about $\mathbf{1 0 0}$ times stronger than the strongest steel. It conducts heat and electricity efficiently and is nearly transparent. Researchers have identified the bipolar transistor effect, ballistic transport of charges and large quantum oscillations in the material.
\end{abstract}

Scientists have theorized about graphene for decades. It has likely been unknowingly produced in small quantities for centuries, through the use of pencils and other similar applications of graphite. Since the discovery, there are only a few graphene-based products that have reached the market, such as the tennis racket by Head, the battery strap by Vorbeck, the oildrilling mud by Nanochem or the phone touch screen by Samsung. These products cannot represent full commercial graphene products. This paper would discuss the operations management side of graphene and graphene related materials. This paper hopes to give an insight of the difficulties in producing graphene.

\section{Keywords—graphene; production; manufacturing; design}

\section{INTRODUCTION}

For many years graphene was deemed a "super" material where its monolayer structure of carbon atoms as shown in figure 1. It was treated solely as a property of various carbonbased materials such as graphite, fullerenes, and carbon nanotubes $[1,2,3]$. The initial works by Geim and Novoselov $[4,5]$ showed the isolation of astonishingly thin carbon films and eventually monolayer graphene by simply using scotch tape and graphite.

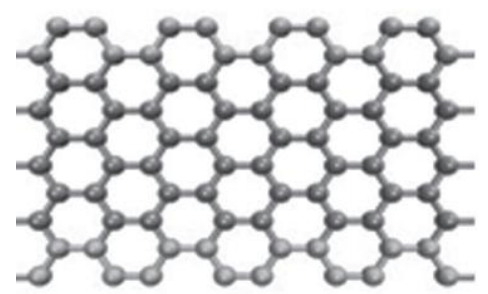

Fig. 1 Graphene structure
Since its discovery, the variety of physical phenomena explored using graphene has expanded at a remarkably fast pace inspiring a wide variety of novel technological applications. Graphene is occupying a centerpiece position in many scientific advances that can change our way of making and using technology. The production processes that could meet the high variety of demand for graphene are still under development. The choice of operations will depend on the required graphene properties and the targeted application, volume requirements and cost needs.

The paper will be structured as the following: the following chapter will provide a review of the term "graphene". Then the paper will discuss the operations management aspect of graphene product. Finally the paper will discuss the difficulties and promises of graphene in product development.

\section{LITERATURE REVIEW}

The term graphene first appeared in 1987[6] to describe single sheets of graphite as a constituent of graphite intercalation compounds; conceptually a GIC is a crystalline salt of the intercalant and graphene. The term was also used in early descriptions of carbon nanotubes [7], as well as for epitaxial graphene [8] and polycyclic aromatic hydrocarbons [9]. Graphene can be considered an "infinite alternant" polycyclic aromatic hydrocarbon [PAH] [10].

Geim [11] defined "isolated or free-standing graphene" as "graphene is a single atomic plane of graphite, which - and this is essential - is sufficiently isolated from its environment to be considered free-standing." Other forms of graphene, such as graphene grown on various metals, can become free-standing if, for example, suspended or transferred to silicon dioxide [SiO2] or silicon carbide.

Andre Geim and Konstantin Novoselov [4] initially used adhesive tape to split graphite into graphene. Achieving single layers typically requires multiple exfoliation steps, each producing a slice with fewer layers, until only one remains. After exfoliation the flakes are deposited on a silicon wafer. Crystallites larger than $1 \mathrm{~mm}$ and visible to the naked eye can be obtained.

The steep rise in graphene application patents shows an increase industrial graphene market in the upcoming Fig. 2 shows the comparison of the patent of different materials. 


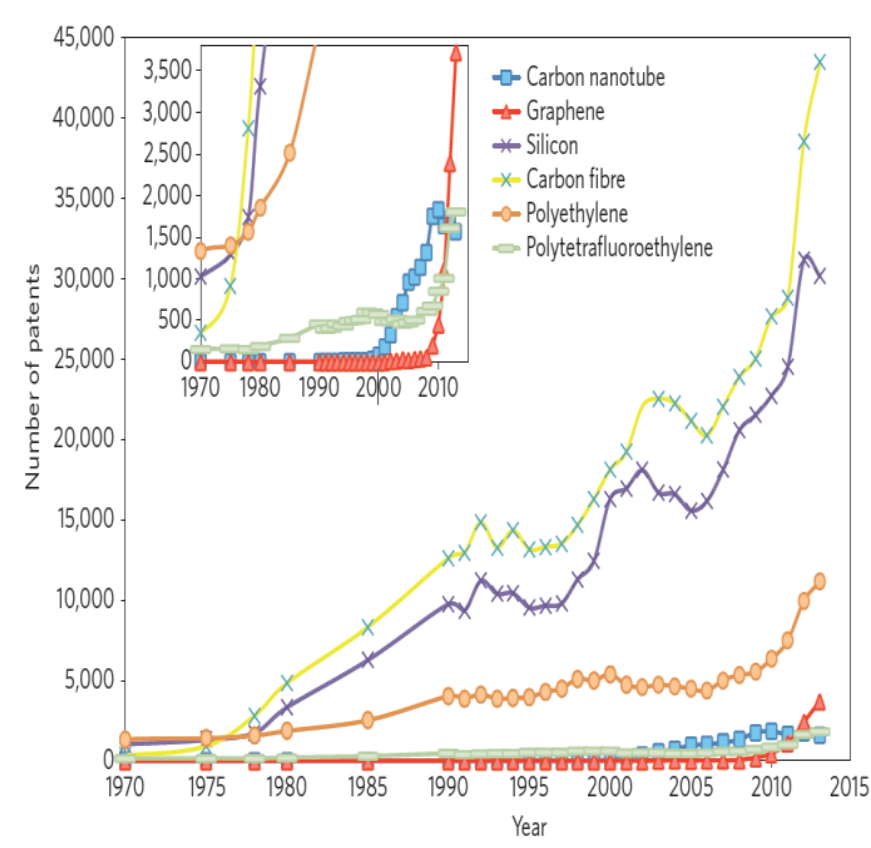

Fig. 2 Number of patent applications and granted patents [12]

Progress in the commercialization of graphene can be assessed by comparing the demand-driven graphene against carbon fibers, carbon nanotubes, silicon, etc. The time of the first step-increase is around 1970 for carbon fibres, around 2001 for carbon nanotubes and around 2009 for graphene. The production of carbon fibres reached 4,600 tonnes per annum in 1978 [13], that is, within 8 years from time zero; the production of carbon nanotubes reached 4,600 tpa in 2011[14].

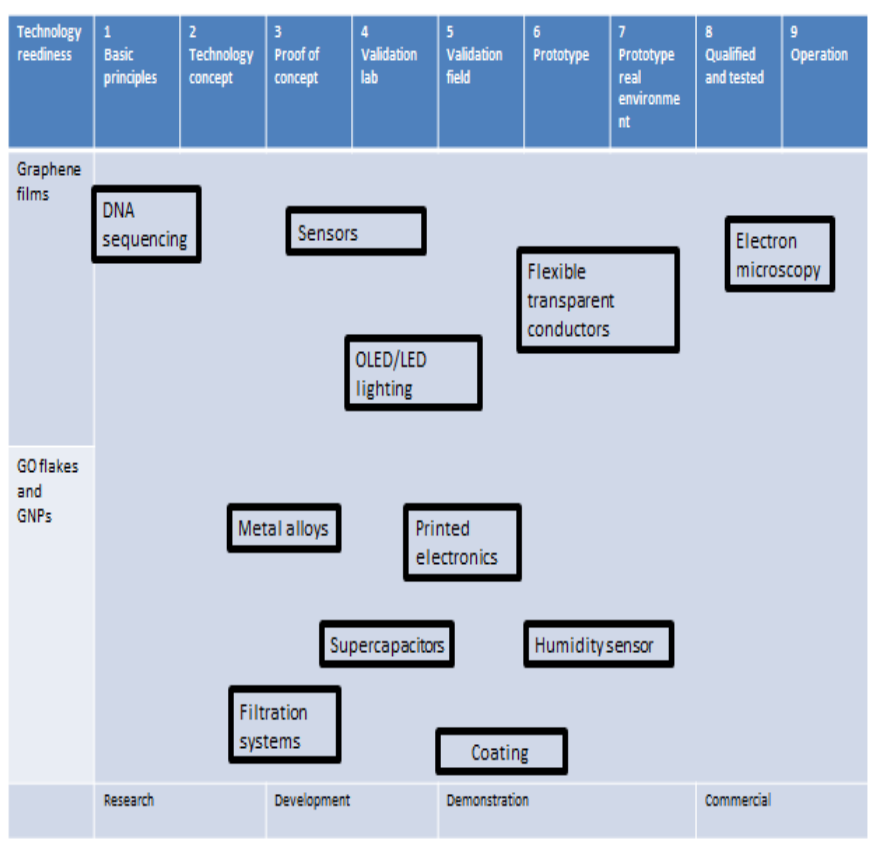

Fig. 3 Graphene applications

Fig. 3 provides a high-level overview of the wide range of applications envisaged for graphene films and GO or graphene flakes. Graphene can be used as a transparent and flexible conductor that holds great promise for various material/device applications, including solar cells, light-emitting diodes [LED], touch panels and smart windows or phones'

\section{OPERATIONS MANAGEMENT ASPECT OF GRAPHENE}

Central to understanding the processes perspective for graphene is the idea that all processes transform inputs into outputs. Figure 4 shows the general transformation process model that is used to describe the nature of processes. Put simply, processes take in a set of input resources, some of which are transformed into outputs of products and some of which do the transforming.

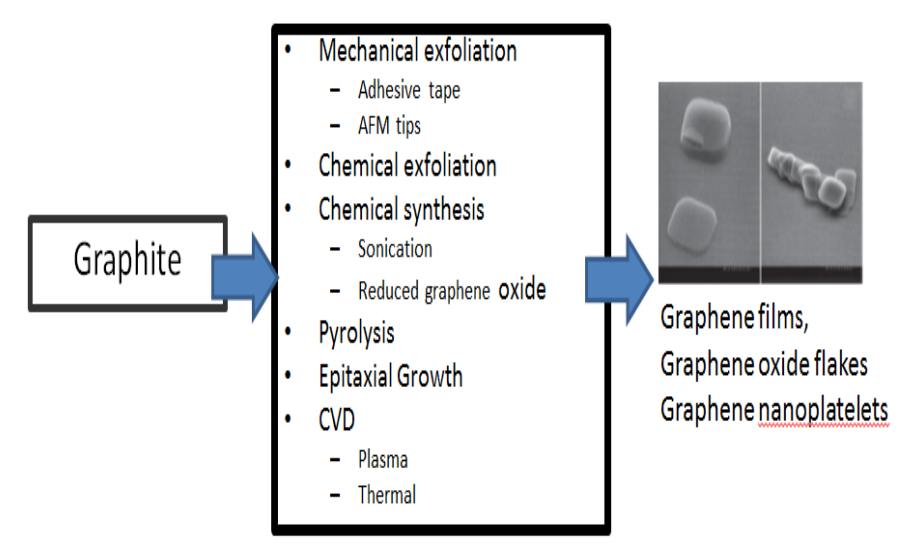

Fig. 4 Transformation process

Several techniques have been established for graphene synthesis. However, mechanical cleaving [exfoliation] [4], chemical exfoliation [15, 16], chemical synthesis [17], and thermal chemical vapor deposition [CVD] [18] synthesis are the most commonly used methods today. Some other techniques are also reported such as unzipping nanotube [19; 20] and microwave synthesis [21]; however, those techniques need to be explored more extensively.

Mechanical exfoliation, shown in Fig. 5, is using scotch tape was the first method of fabricating graphene with different numbers of layers. This technique is simple and low cost, but the control of large-scale synthesis and reproducibility of the same structure is yet to be demonstrated. Mechanical cleaving of highly ordered pyrolytic graphite [HOPG] by atomic force microscopy [AFM] tips was first developed in order to fabricate graphene from a few layers down to a mono-atomic single layer [22]. Mechanical exfoliation of graphene is produced by high-velocity clusters impacting on a graphite surface [23]. The graphene nanoribbon produced by this method was $\sim 30 \mathrm{~nm}$ thick.

Research indicated graphene exfoliated from microwave irradiation of graphite-intercalated compounds in a solution process followed by combining those exfoliated sheets with carbon nanotubes [CNTs] [24 and 25]. They claimed that the graphene CNT combined sheet resistance was $181 \mathrm{ohm} / \mathrm{sq}$ with 
$82.2 \%$ transmittance, which is equivalent to the commercially available indium tin oxide [ITO].

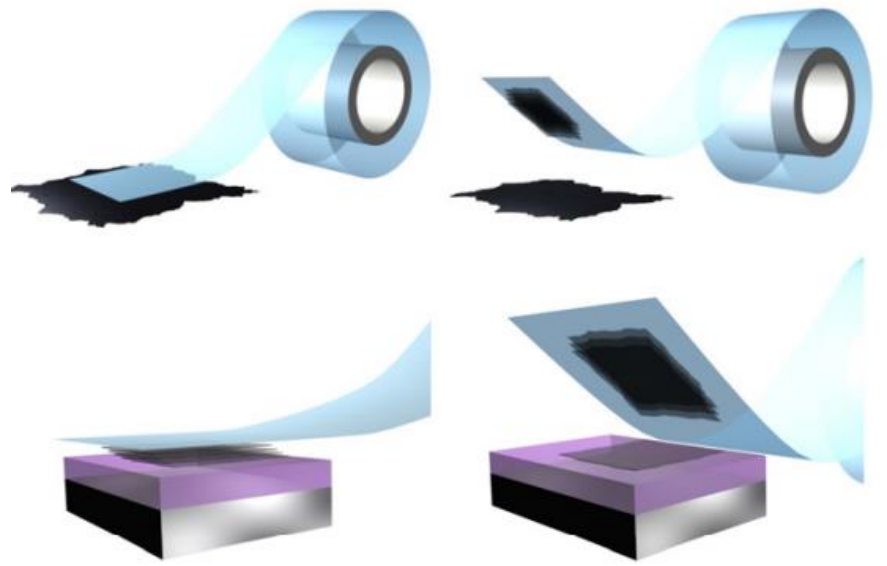

Fig. 5 Mechanical exfoliation of Graphene [26]

Plasma-assisted etching of graphite to form multi-layered graphene and monolayer graphene was also demonstrated in another report [27]. This is another top-down approach that involves the gradual thinning process of graphite to graphene using plasma in an $\mathrm{H} 2$ and $\mathrm{N} 2$ atmosphere. In a different approach, de Parga et al. reported the epitaxial graphene formation on $\mathrm{Ru}$ [0001] under ultrahigh vacuum [UHV] conditions [ 10-11 Torr] [28].

Chemical synthesis was manifested in low temperature, large-scale graphene synthesis methods, which are transfer free processes and capable of fabricating graphene film on any substrate, as shown in Figure 6. Solution process synthesis methods are advantageous for easy fabrication of functionalized graphene. Nevertheless, the graphene produced using these techniques is defective, partially reduced graphene oxide, which seriously compromises the physical properties of graphene.

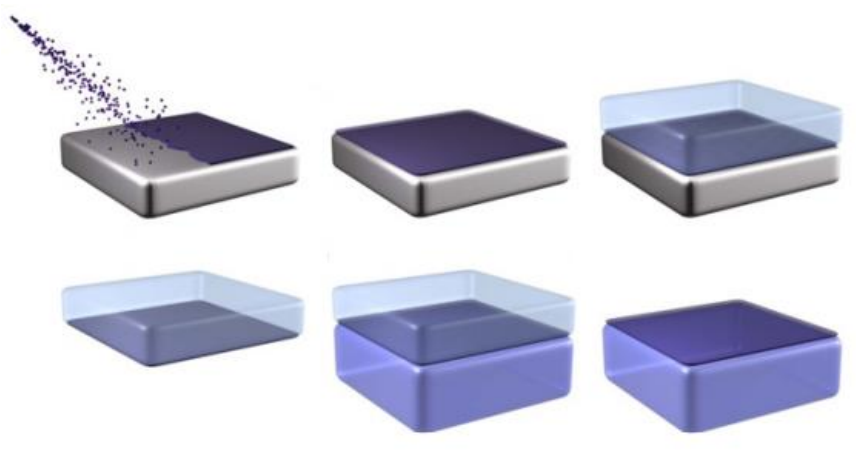

Fig. 6 Chemical vapor deposition (CVD) [26]

Researcher reported the metal catalyzed formation of graphene using amorphous carbon at high temperature [29]. Thermal chemical vapor deposition has been proven to be a more industrially feasible and scalable which has possibilities to obtain various morphologies of graphene over a wide range of substrates. However, the process is costly and incapable of producing graphene directly on polymer substrates, and involves a transfer process that could introduce defects and contaminants in graphene.

Operations add value for customers and contribute to competitiveness by being able to satisfy the requirements of its customers [30]. There are five aspects of operations performance, all of which to a greater or lesser extent will affect customer satisfaction and business competitiveness.

- Quality - doing things right, providing error-free goods and services that are 'fit for their purpose'. Example would be to obtain valuable control over the number of graphene layers produced along with homogeneous electrical and optical properties.

- Speed - minimizing the time between a customer asking for goods and services and the customer receiving them in full. The speed of industrial uptake of graphene will be influenced by factors such as the optimization of supply-chain logistics.

- Dependability - doing things on-time, keeping the delivery promises that have been made to customers. For graphene, the required knowledge and understanding to provide atomically precise fabrication of this material, in a reproducible manner, that is compatible with current semiconductor technology is still lacking.

- Flexibility - the ability to vary or adapt the operation's activities to cope with unexpected circumstances. For example epitaxial growth on a $\mathrm{SiC}$ surface yields high-quality, highpurity graphene that exhibits good electrical properties. However, due to lack of flexibility on different substrates, its application in a wide range of electronic and optoelectronic devices is seriously limited

- Cost - doing things cheaply, producing goods at a cost that enables them to be priced appropriately for the market while still allowing a return to the organization

The priorities among these operations' sub-goals and their relative emphases should be direct reflections of the organization's mission. Relating these six operations sub-goals to the broader strategic choices above, it is clear that quality, efficiency, and dependability [customer service] are reflected in the sub-goals.

Flexibility encompasses adaptability but also relates to product/service and process characteristics: Once choices about product and process are made, boundaries for meeting the other operations objectives are set.

According to [12] limited material availability can potentially slow down the commercial adoption of graphene. Thus currently the cost is medium important. The flexibility has to be high so the variety of product can be researched. The focus of graphene manufacturers is therefore rapidly shifting towards improving batch-to-batch consistency and costs. 


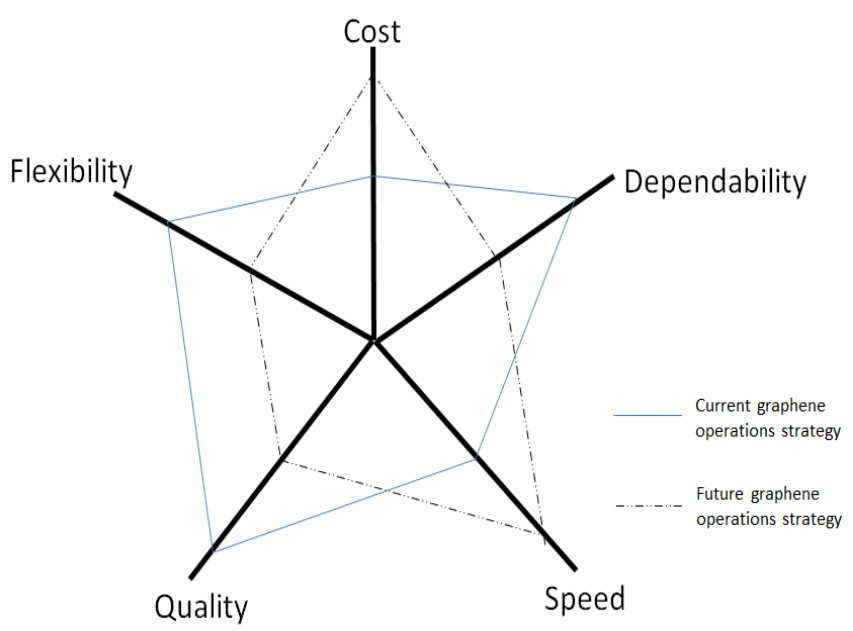

Fig. 7 A polar representation

A polar representing of the relative importance of performance objectives for a graphene product is shown in Fig. 7. The scales which represent the importance of each performance objective have the same origin. A line describes the relative importance of each performance objective. The closer the line is to the common origin, the less important is the performance objective to the operation.

Quality, speed, dependability and flexibility can improve cost performance. But externally this is not always the case. There is a 'trade-off' between performance objectives [30]. Improving the performance of one performance objective might only be achieved by sacrificing the performance of another. An operation might wish to improve its cost efficiencies by reducing the variety of products or services that it offers to its customers.

Costs have already come down considerably since graphene was first introduced in the research community. The material cost required to enable commercialization is also application dependent, and a higher initial material cost is likely to promote adoption in applications that exploit multiple graphene properties.

A slower decrease in the cost of graphene will delay the deployment in price sensitive, large-volume products and in applications looking at graphene for material replacements, cost savings or medium level improvement in a single performance parameter. Consistency is crucial for industrial applications and depends on process repeatability. Thus the future of the graphene research should focus on increasing importance of speed.

Given the diverse ranges of graphene materials, an effort to standardize the definition of different types of graphene and related materials will accelerate the process of adoption and commercialization of graphene by reassuring potential adopters about the nature and quality of these materials.

\section{CHALLENGES}

The toxicity of graphene has been extensively debated in the literature. The most comprehensive review on graphene toxicity [31] Results show that the toxicity of graphene is dependent on several factors such as shape, size, purity, postproduction processing steps, oxidative state, functional groups, dispersion state, synthesis methods, route and dose of administration, and exposure times.

Research at Stony Brook University showed that graphene nanoribbons, graphene nanoplatelets and graphene nanoonions are non-toxic at concentrations up to $50 \mu \mathrm{g} / \mathrm{ml}$. These nanoparticles do not alter the differentiation of human bone marrow stem cells towards osteoblasts [bone] or adipocytes [fat] suggesting that at low doses graphene nanoparticles are safe for biomedical applications [32 and 33].

All production processes and chemical treatment may introduce structural defects in graphene, such assingle and multiply vacancies, dislocation like defects, carbon adatoms, or accessory chemical groups [33 and 34]. The underlying strengthening and toughening mechanisms of graphenepolymer nanocomposites is still not well understood and the influence of defective graphene on them. Further study is much required [35].

Due to the nano-scale dimensions, it is difficult to accurately evaluate the properties of graphene sheets via experiment [36]. Deformation and failure of graphene sheet and graphene-polymer interface is dependent on both structural defects and temperature [37].

There are needs to control individual features in graphene devices accurately enough to provide sufficient reproducibility in their properties. The latter is exactly the same challenge that the Si technology has been dealing with successfully. Currently proof-of-principle nm-size graphene devices can be made by electrochemical etching using scanning-probe nanolithography [38].

\section{$\mathrm{V}$ CONCLUSION}

Graphene exhibits several unusual physical properties that can be applicable to future electronic and optoelectronic devices. From synthesis routes to its growth mechanism, or from its eccentric properties to possible applications, the subject is still under scholarly debate.

Graphene synthesis processes have advantages and disadvantages focusing on its field of application. Finally, this is a growing field of science, which needs more rigorous studies to obtain high quality graphene by controlling over the process parameters and more comprehensible scientific understanding. The restricted graphene mass-production and limited reproducibility in device performances are still important matters that researchers should consider in order to push graphene-based technology into marketing and sale. 


\section{REFERENCES}

[1] R. Peierls, Quelques properties typiques des corpes solides. Annales d' Institut Henri Poincare 5: 177, 1935.

[2] L. Landau, Zur Theorei der phasenumwandlugen II. Physikalische Zeitschrift Sowjetunion 11: 26, 1937.

[3] N. D. Mermin, "Crystalline order in two dimensions". Physical Review 176: $250,1968$.

[4] K. S. Novoselov, A. K. Geim, S. V. Morozov, D. Jiang, Y. Zhang, S. V. Dubonos, I. V. Grigorieva and A. A. Firsov, "Electric field effect in atomically thin carbon films", Science 306: 666, 2004.

[5] K. S. Novoselov, A. K. Geim, S. V. Morozov, D. Jiang, M. I. Katsnelson, I. V. Grigorieva, S. V. Dubonos, and A. A. Firsov, "Twodimensional gas of massless Dirac fermions in graphene". Nature 438: 197-200, 2005.

[6] S. Mouras, et al., "Synthesis of first stage graphite intercalation compounds with fluorides", Revue de Chimie Minérale. 24: 572, 1987.

[7] R. Saito, M. Fujita, G. Dresselhaus, M. Dresselhaus, "Electronic structure of graphene tubules based on C60", Physical Review B. 46 [3]: 1804-1811, 1992. Bibcode:1992PhRvB..46.1804S. doi:10.1103/PhysRevB.46.1804.

[8] I. Forbeaux, J. M. Themlin, J. M. Debever, "Heteroepitaxial graphite on $6 \mathrm{H}-\mathrm{SiC}[0001]$ : Interface formation through conduction-band electronic structure", Physical Review B. 58 [24]: 16396-16406, 1998.

[9] S. Wang, S. Yata, J. Nagano, Y. Okano, H. Kinoshita, H. Kikuta, and T. Yamabe, "A new carbonaceous material with large capacity and high efficiency for rechargeable Li-ion batteries", Journal of the Electrochemical Society. 147 [7]: 2498, 2000.

[10] C. D. Simpson, J. D. Brand, A. J. Berresheim, L. Przybilla, H. J. Räder, and K. Müllen, "Synthesis of a Giant 222 Carbon Graphite Sheet". Chemistry. 6 [6]: 1424-1429, 2002.

[11] A. Geim, "Graphene: Status and Prospects", Science. 324 [5934]: 15304, 2009.

[12] A. Zurutuza and C. Marinelli, "Challenges and opportunities in graphene commercialization” Nature Nanotechnology, Vol 9, October.730-734, 2014.

[13] G. Owen, The Rise and Fall of Great Companies: Courtaulds and the Reshaping of the Man-Made Fibres Industry [Oxford Univ.Press/Pasold Research Fund, 2010.

[14] M. F. L. De Volder, S. H. Tawfick, R. H. Baughman, \& A. J. Hart, Science 339, 535-539, 2013

[15] M. J. V. C. Allen, C. Tung, and R. B. Kaner, Honeycomb carbon: A review of graphene. Chemical Reviews 110 [1]:132-145, 2010.

[16] L. M. Viculis, J. M. Julia and B. K. Richard, "A chemical route to carbon nanoscrolls", Science 299 [5611]:1361, 2003.

[17] S. Park and R. S. Ruoff, "Chemical methods for the production of graphenes", Nature Nanotechnology 4 [4]:217-224, 2009.

[18] A. S. Reina, S. Thiele, X. T. Jia, et al., "Growth of large-area single- and bi-layer graphene by controlled carbon precipitation on polycrystalline Ni surfaces", Nano Research 2 [6]:509-516, 2009.

[19] L. Y. Jiao, X. R. Wang, G. Diankov, H. L. Wang, and H. J. Dai, "Facile synthesis of highquality graphene nanoribbons", Nature Nanotechnology 5 [5]:321-325, 2010.

[20] D. V. Kosynkin, A. L. Higginbotham, A. Sinitskii, et al., Longitudinal unzipping of carbon nanotubes to form graphene nanoribbons. Nature 458 [7240]:872-876, 2009.
[21] G. Q. Xin, W. Hwang, N. Kim, S. M. Cho, and H. Chae, "A graphene sheet exfoliated with microwave irradiation and interlinked by carbon nanotubes for high-performance transparent flexible electrodes", Nanotechnology 21 [40], 2010.

[22] X. K. Lu, M. F. Yu, H. Huang and R. S. Ruoff, Tailoring graphite with the goal of achieving single sheets. Nanotechnology 10 [3]:269-272, 1999.

[23] A. N. Sidorov, T. Bansal, P. J. Ouseph, and G. Sumanasekera, "Graphene nanoribbons exfoliated from graphite surface dislocation bands by electrostatic force", Nanotechnology, 2011.

[24] G. Q. Xin, W. Hwang, N. Kim, S. M. Cho, and H. Chae, "A graphene sheet exfoliated with microwave irradiation and interlinked by carbon nanotubes for high-performance transparent flexible electrodes", Nanotechnology 21 [40], 2010.

[25] V. Sridhar, J. H. Jeon and I. K. Oh, "Synthesis of graphene nano-sheets using ecofriendly chemicals and microwave radiation", Carbon 48. [10]:2953-2957, 2010.

[26] K. S. Novoselov and N. A. H. Castro, "Two-dimensional crystals-based heterostructures: materials with tailored properties", Phys. Scr. T1461-3, 2012.

[27] K. S. Hazra, et al., "Thinning of multilayer graphene to monolayer graphene in a plasma environment", Nanotechnology 22 [2]:025704, 2011.

[28] A. L. V. de Parga, F. Calleja, B. Borca, et al., "Periodically rippled graphene: Growth and spatially resolved electronic structure", Physical Review Letters 100 [5], 2008

[29] M. Zheng, K. Takei, B. Hsia, et al., "Metal-catalyzed crystallization of amorphous carbon to graphene", Applied Physics Letters 96 [6], 2010.

[30] N. Slack, S. Chambers, R. Johnston and A. Betts, Operations Management, Person education London, 2014

[31] G. Lalwani, M. D'Agati, ; A. M. Khan, B. Sitharaman, "Toxicology of graphene-based nanomaterials". Advanced Drug Delivery Reviews, 2016.

[32] Y. Talukdar, J. T. Rashkow, G. Lalwani, S. Kanakia, B. Sitharaman, "The effects of graphene nanostructures on mesenchymal stem cells", Biomaterials. 35 (18): 4863-77, 2014

[33] Y. Li, H. Yuan, B. A. von Dem, M. Creighton, R. H. Hurt, A. B. Kane, H. Gao, "Graphene microsheets enter cells through spontaneous membrane penetration at edge asperities and corner sites", Proceedings of the National Academy of Sciences. 110 (30): 12295-12300, 2013.

[34] V. Singh, D. Joung, L. Zhai, S. Das, S. I. Khondaker and S. Seal, Graphene based materials: Past, present and future, Prog Mater Sci, 56 1178, 2011.

[35] M. Wang, C. Yan, N. Hu, "Deformation and Failure of Graphene Sheet and Graphene-Polymer Interface", 13th International Conference on Fracture, June 16-21, Beijing, China, 2013.

[36] M. A. Rafiee, J. Rafiee, I. Srivastava, Z. Wang, H. H. Song, Z. Z. Yu and N. Koratkar, "Fracture and Fatigue in Graphene Nanocomposites", Small, 6, 179, 2010

[37] J. Lee, V. Varshney, J. S. Brown, A. K. Roy and B. L. Farmer, "Single mode phonon scattering at carbon nanotube-graphene junction in pillared graphene structure", Appl Phys Lett, 100 183111, 2012.

[38] Tseng, A.A., Notargiacomo A., Chen T.P. Nanofabrication by scanning probe microscope lithography: A review. J. Vac. Sci. Tech. B 23, 877894 (2005). 